\title{
Capacity, Throughput, and Delay Analysis of a Cellular DS CDMA System With Imperfect Power Control and Imperfect Sectorization
}

\author{
Michel G. Jansen, Student Member, IEEE, and Ramjee Prasad, Senior Member, IEEE
}

\begin{abstract}
An analytical model is developed to evaluate the performance of a cellular slotted DS CDMA system in terms of user capacity, throughput, and delay for the reverse link, i.e., from mobile to base station, considering interference from both home cell and adjacent cells. The user capacity is studied for voice communications and the throughput and delay are investigated for data communications. The effect of both imperfect power control and imperfect sectorization on the performance is investigated. It is shown that the system is rather sensitive to small power control errors and that voice activity monitoring and sectorization are good methods to improve the performance of cellular DS CDMA systems.
\end{abstract}

\section{INTRODUCTION}

\section{A} MONG SEVERAL TYPES of Code Division Multiple Access (CDMA), Direct Sequence CDMA (DS CDMA) is a strong candidate for future personal communications because this technique offers inherent multipath diversity and robustness to varying channel conditions [1]-[4]. In order to obtain a reasonable performance using DS CDMA systems, additional considerations are required. Three techniques proposed in the literature to increase the performance of a DS CDMA system are power control in order to combat the nearfar effect; sectorization; and voice activity monitoring in order to decrease the interference power detected at the receiving antenna. In practice power control errors occur and due to antenna imperfections, sectorization is not always perfect.

In this paper a general analytical model is presented that can be used to evaluate the influence of both imperfect power control and imperfect sectorization on the performance of two types of CDMA systems, namely voice-oriented and dataoriented systems. For the first type, the maximum user capacity is an important performance measure while for the second one, throughput and delay characteristics are appropriate performance parameters. An analytical model is developed assuming that the data-oriented system is slotted and simultaneous transmission of two or more packets is permitted. If the actual number of transmitting packets exceeds the value of the maximum capacity, then all packets are destroyed. In both systems only the reverse link, i.e., from mobile to base station is considered.

Manuscript received January 11. 1994; revised March 9, 1994; accepted April 22, 1994.

The authors are with the Telecommunications and Traffic Control Systems Group, Delft University of Technology. $2600 \mathrm{GA}$ Delft, The Netherlands.

IEEE Log Number 9407341
In Section II the principles of CDMA are discussed briefly and the performance enhancing techniques mentioned above are described. In Sections III and IV, respectively, the models for the capacity analysis and the throughput and delay analysis are explained. Section V shows the computational results and, finally, in Section VI conclusions and recommendations are presented.

\section{Cellular DS CDMA}

In a direct sequence spread spectrum system the data signal at the transmitter is multiplied by a pseudo-random user-specific code sequence waveform. At the receiver the original data can be recovered by correlating the received spread spectrum signal with the correct code sequence. If code sequences with good correlation properties are used, after the correlation operation the unwanted signals appear as noise-like signals with a very low power density spectrum. In order to establish the spreading effect, the duration $T_{c}$ of a code symbol (chip) must be chosen much smaller than the duration $T_{b}$ of a data symbol. Therefore the bandwidth $B_{\mathrm{ss}}$ of the transmitted spread spectrum signal is much larger than the bandwidth $B_{s}$ of the original data signal. The ratio between transmitted and original bandwidth is defined as the processing gain:

$$
\mathrm{PG} \stackrel{\wedge}{=} \frac{B_{\mathrm{ss}}}{B_{s}}
$$

In practice, this number depends on the specific modulation method. We will however use the following approximation:

$$
\mathrm{PG} \triangleq \frac{B_{\mathrm{ss}}}{B_{s}} \approx \frac{T_{b}}{T_{c}}
$$

where $T_{b}$ is the bit duration and $T_{c}$ is the chip duration.

Since two code sequences with a relative delay of more than two chip times usually have a low correlation value compared to the fully synchronized situation, DS CDMA offers the possibility to distinguish between paths with a relative delay of more than two chip times. This is called the inherent diversity of DS CDMA, implying that it is possible to resolve a number of paths separately using only one receiver. This property makes DS CDMA suitable for applications in mobile radio environments that are usually corrupted with severe multipath effects.

It is well known that one of the most serious problems faced by a DS CDMA system is the multi-user interference. Because all users are transmitting in the same frequency band and 
the crosscorrelations of the codes are rarely zero, the signalto-interference ratio and hence the performance decreases as the number of users increases, which shows that DS CDMA is an interference-limited rather than a noise-limited system. The near-far effect plays an especially important role when considering multi-user interference. The near-far effect can be explained by considering the reverse link. Due to the pathloss law (which implies that the received power decreases as the transmitter-receiver distance increases), a close user will dominate over a user located at the cell boundary. The pathloss law is usually described by assuming that the received power $P_{r}$ is proportional to the distance $d$ to the power $\beta$ :

$$
P_{r} \sim d^{-\beta}
$$

where $\beta$ is the path-loss law exponent. In the case of free space propagation, the value of $\beta$ is 2 . In a practical mobile radio environment the value of $\beta$ is in the range of 3 to 5 , which is caused by the fact that the radio waves are reflected and partially absorbed by objects between receiver and transmitter and by the surface of the earth. In order to combat the nearfar effect, power control can be used. There are two other important techniques to reduce the multi-user interference in a cellular DS CDMA system. The first one is sectorization, which is established by using sectorized antennas at the base station, implying that only interference signals are received from within a limited angle. The second important technique is voice activity monitoring. A system using voice activity monitoring prevents a mobile from radiating power during speech pauses. A complication in a cellular system is the fact that a base station not only receives interference from users within its own cell (intra-cell interference) but also from users in other cells (inter-cell interference).

\section{A. Power Control}

Power control can be established by letting the base station continuously transmit a (wideband) pilot signal that is monitored by all mobile terminals. According to the power level detected by the mobile, the mobile adjusts its transmission power. Hence mobiles near the cell boundary transmit at a lower power level than mobiles located close to the base station. This is open loop power control. It is also possible to use closed loop power control as is suggested in [5] and [6]. In this case the base station measures the energy received from a mobile and controls the transmit power of the mobile by sending a command over a (low data rate) command channel. In a practical power control system, power control errors occur, implying that the average received power at the base station may not be the same for each user signal.

The performance of a power control system depends on the power control algorithm, speed of the adaptive power control system, dynamical range of the transmitter, spatial distribution of users, and propagation statistics (such as fading and shadowing). All these factors influence the probability density function (pdf) of the received power. Since the objective here is to investigate the influence of power control imperfections on the system performance, the explicit influence of the factors mentioned above on the pdf of the average received power is not investigated and it can be a subject for future study.

Investigations on DS CDMA have shown that the pdf of the received power $P$ due to the combined influence can be assumed to be $\log$ normal [1], [2].

$$
f(P)=\frac{1}{\sqrt{2 \pi} \sigma P} \exp \left[-\frac{\ln ^{2}(P)}{2 \sigma^{2}}\right]
$$

where the imperfection in the power control system is determined by the logarithmic standard deviation $\sigma$ of the lognormal power distribution of the received signal. In the case of perfect power control the logarithmic standard deviation is $0 \mathrm{~dB}$.

There are a number of motivations for choosing a lognormal distribution for the individual received power. First, the received power at the base station depends on a lot of independent factors. It can be assumed that each factor gives a contribution in $\mathrm{dB}$ to the received power. Using the central limit theorem it is clear that the logarithm of the received power is Gaussian-distributed, implying that the received power is lognormally distributed. The second motivation is that this model provides a means to investigate the influence of imperfect power control analytically. A third is data provided in [5], [6], where it is shown that the received signal to noise ratio is log-normally distributed with standard deviation between 1 and $2 \mathrm{~dB}$. Assuming constant noise power, this implies that the received power can be modeled as a lognormal variable.

If a number of $k$ mobiles is transmitting and if the power of each mobile is controlled individually, then the total received interference power $P_{I}$ is the summation of $k$ independent identically log-normally distributed random variables denoted by $P_{i}$ :

$$
P_{I}=\sum_{i=1}^{k} P_{i} .
$$

Fenton [10] showed that the pdf of $P_{I}$ for $k$ users is approximately log-normal with the following logarithmic mean $m_{I}(k)$ and logarithmic variance $\sigma_{I}^{2}(k)$ :

$$
\begin{gathered}
\sigma_{I}^{2}(k)=\ln \left(\frac{1}{k} e^{\sigma^{2}}+\frac{k-1}{k}\right) \\
m_{I}(k)=\ln (k)+m+\frac{\sigma^{2}}{2}-\frac{1}{2} \ln \left(\frac{k-1}{k}+\frac{1}{k} e^{\sigma^{2}}\right) .
\end{gathered}
$$

This method is valid for a logarithmic standard deviation $\sigma$ less than $4 \mathrm{~dB}$.

\section{B. Sectorization}

The multi-user interference received at the base station can be reduced by dividing a cell into a number of sectors by means of directional antennas. In the case of perfect directional antennas there is a sharp separation between the sectors. Due to overlap and sidelobe anomalies of practical antennas the base station still receives some interference from users in other sectors. This effect can be studied by modeling 


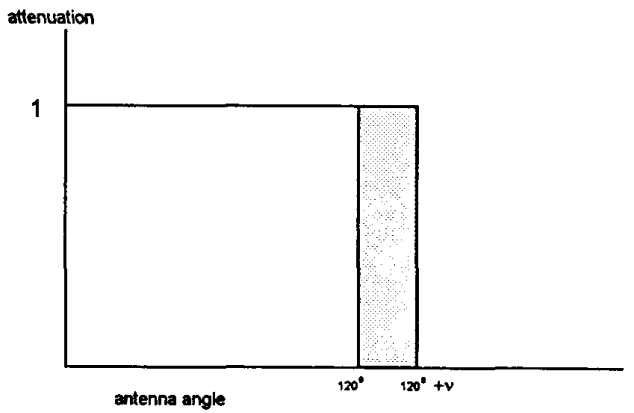

(a)

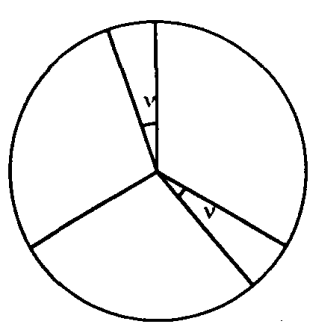

(b)

Fig. 1. (a) Radiation pattern model for imperfect directional antenna with opening angle $120^{\circ}$. (b) Sector coverage with an imperfect directional antenna with opening angle $120^{\circ}$

the real antenna gain pattern for instance by a parabolic function. For simplicity, however, we assume the simplified antenna radiation pattern shown in Fig. 1(a) and (b) where the antenna imperfections are modeled by the overlap angle $v$. It is suggested to transform the real antenna gain patterns to this uniform model, analogously as the method for describing noise by the noise equivalent bandwidth. If $D$ is the number of sectors and $v$ is the overlap angle, then a relation can be derived between the ratio of the total interference power received in a sectorized system and the total interference power received in a non-sectorized system denoted by $F_{s}$.

$$
F_{s} \triangleq \frac{\tilde{P}_{\text {sectorized }}}{\tilde{P}_{\text {non-sectorized }}}=\left(\frac{1}{D}+\frac{2 v}{360}\right)
$$

where $D$ is an integer. From (8) it is clear that $v=0$ corresponds with perfect sectorization and the combination $D=1$ and $v=0$ corresponds to the situation without sectorization.

\section{Voice Activity Monitoring}

Voice activity monitoring implies that the transmitter is not active during silent periods in human speech. It is possible to detect a silent period in the speech signal and let the transmitter stop transmitting during this period. Voice activity factors between $35 \%$ and $40 \%$ are reported in [3] and [4]. Studies done in Europe suggest that the total activity due to voice and background noise is higher in a mobile environment than in a wireline system and can have values between 50 and $60 \%$ [7]. When using voice activity monitoring, the probability that $k$ out of $n$ interferers are active can be described by a binomial

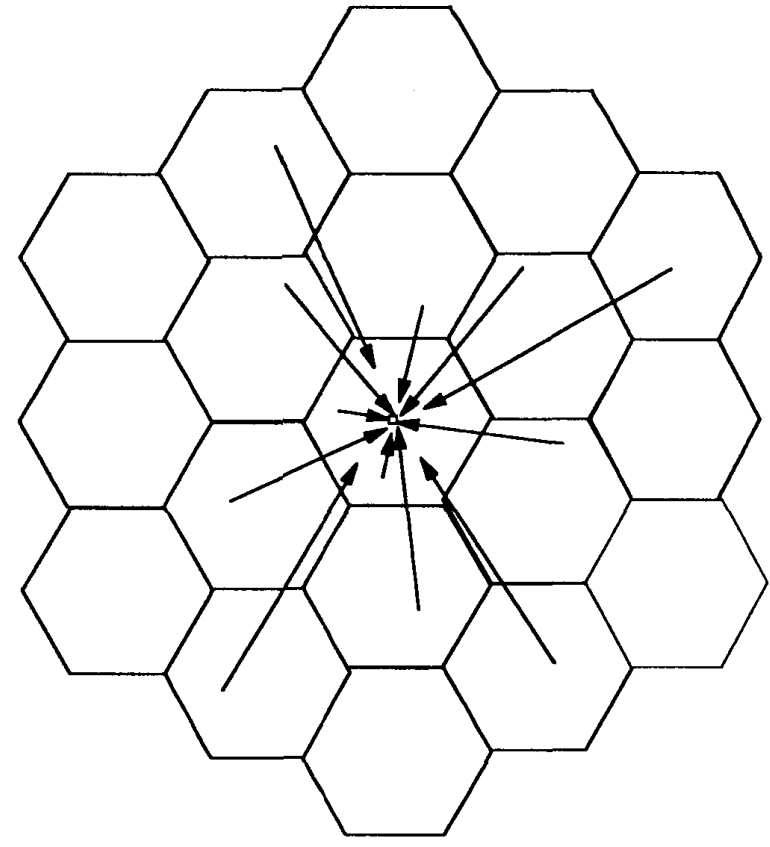

Fig. 2. Inter-cell interference in a cellular system.

distribution:

$$
P(n, k)=\left(\begin{array}{l}
n \\
k
\end{array}\right) a^{k}(1-a)^{n-k}
$$

where $a$ is the voice activity factor. The effect of the voice activity factor on the CDMA capacity is investigated in this paper.

\section{Inter-Cell Interference}

In a cellular system, each base station not only receives interference from mobiles in the home cell (intra-cell interference) but also from terminals located in adjacent cells (Fig. 2). This kind of interference is called inter-cell interference. The principle of the CDMA protocol allows each cell to use the same frequency band, removing the need for a mobile to change its frequency when moving into another cell (soft handover). In order to model the interference received from terminals in other cells, we investigate here the interference power received by the base station in the home cell $(H)$ from mobile terminals in an other cell $(O)$ with base station separation distance $d$ (Fig. 3).

We have adopted a generalized version of the approach as described in [9]. For analytical convenience the hexagonal cell structure is approximated by circles with radius $R$. The power of terminals in cell $H$ and cell $O$ is controlled by the base station in $H$ and $O$, respectively. In the case of perfect power control, the power received by a base station from each mobile terminal in its service area is constant. This power is denoted by $S_{p}$. We assume that users are uniformly distributed in each cell with user density

$$
\eta=\frac{N}{\pi R^{2}}
$$




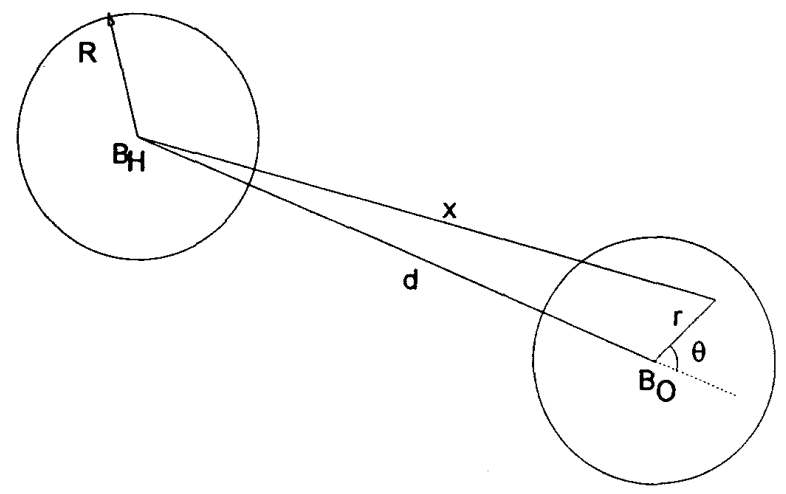

Fig. 3. Interference from terminals in a distant cell.

where $N$ is the number of users per cell and $R$ is the cell radius. The power control strategy is such that a terminal at a distance $r$ from the base station transmits with power

$$
P_{T}(r)=S_{p} \cdot r^{\beta}
$$

implying that the power received by the base station is

$$
P_{R}(r)=S_{p} \cdot r^{\beta} \cdot r^{-\beta}=S_{p}
$$

where $\beta$ is the path loss law exponent that is two in case of free space loss and lies between three and five for mobile systems. In [9] the special case of $\beta=4$ was considered while we derive an expression which is valid for all values of $\beta$. From Fig. 3 it can be seen that the power received by the base station denoted by $P_{\mathrm{RB}}(d)$ from a mobile users in a cell at distance $d$ is obtained by integrating over the cell area $(A)$ :

$$
P_{\mathrm{RB}}(d)=\int S_{p}\left(\frac{r}{x}\right)^{\beta} \eta d A
$$

where

$$
x=\sqrt{d^{2}+r^{2}+2 d r \cos \theta}
$$

Using Fig. 3 and substituting (10) and (14), (13) can be written as

$$
P_{\mathrm{RB}}(d)=\frac{2 N \cdot S_{p}}{\pi R^{2}} \int_{0}^{R} d r \cdot r^{\beta+1} \int_{0}^{\pi} \frac{d \theta}{\left(d^{2}+r^{2}+2 d r \cos \theta\right)^{\beta / 2}} .
$$

In [6] a closed expression was found for the case $\beta=4$, which we obtain from (15):

$$
\begin{aligned}
P_{R B}(d)=2 \cdot N \cdot S_{p} \cdot & {\left[2\left(\frac{d}{R}\right)^{2} \ln \left(\frac{\left(\frac{d}{R}\right)^{2}}{\left(\frac{d}{R}\right)^{2}-1}\right)\right.} \\
& \left.-\frac{4\left(\frac{d}{R}\right)^{4}-6\left(\frac{d}{R}\right)^{2}+1}{2\left(\left(\frac{d}{R}\right)^{2}-1\right)^{2}}\right] .
\end{aligned}
$$

Considering now a hexagonal cellular structure (Fig. 2), it is possible to compute the total received interference from all the cells in the system. The interference correction factor due to multiple cell interference $F_{m}$ is defined as the ratio of the interference power received from the outer cells $\left(I_{m}\right)$ and the

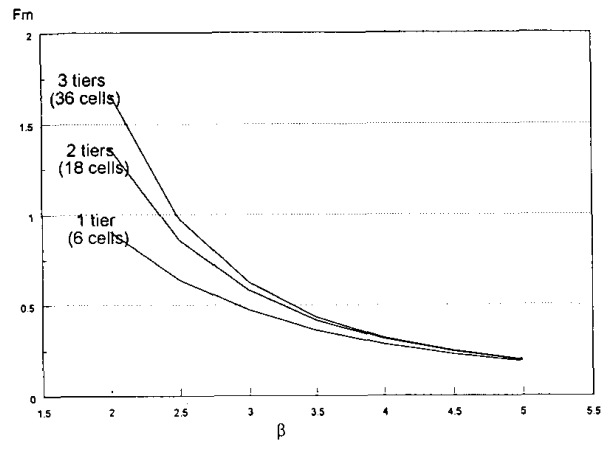

Fig. 4. Multiple cell interference reduction factor $F_{m}$ as function of the path loss law exponent $\beta$ considering different numbers of interfering cells.

TABLE I

\begin{tabular}{|c|l|l|l|l|}
\hline & \multicolumn{4}{|c|}{ Interference correction factor $F_{\mathrm{m}}$} \\
\hline Number of tiers & $\beta=2$ & $\beta=3$ & $\beta=4$ & $\beta=5$ \\
\hline 1 & 0.904 & 0.417 & 0.284 & 0.191 \\
\hline 2 & 1.365 & 0.579 & 0.312 & 0.199 \\
\hline 3 & 1.669 & 0.625 & 0.319 & 0.200 \\
\hline 5 & 2.079 & 0.667 & 0.323 & 0.201 \\
\hline 10 & 2.665 & 0.701 & 0.326 & 0.201 \\
\hline 15 & 3.018 & 0.714 & 0.326 & 0.201 \\
\hline
\end{tabular}

interference power generated by users in the home cell $\left(I_{h}\right)$. Using (12), $F_{m}$ can be written as

$$
F_{m} \triangleq \frac{I_{m}}{I_{h}}=\frac{I_{m}}{(N-1) S_{p}}
$$

where $N$ is the number of users per cell and $S_{p}$ is the power received from a user in the case of perfect power control.

The value of $I_{m}$ depends on the value of $\beta$ and on the number of cell tiers considered. From (15) it follows that for a large number of users $(N \gg 1)$, the ratio $F_{m}$ is a constant because $I_{m}$ is proportional to $N$. Fig. 4 and Table I show the interference correction factor $F_{m}$ as function of the path loss law exponent $\beta$ considering several number of cell tiers around the home cell. It is clear that for larger values of $\beta$, the influence of the outer cell tiers is decreasing with increasing tier number. For $\beta=4$ no significant difference was found between considering 10 or 15 tiers. In both cases, we found $F_{m}=0.326$. This value is less than the value of 0.66 reported in [4] that was obtained by simulation assuming log-normal shadowing. The value 0.326 was found analytically for the situation without shadowing and is confirmed by results presented in [9]. The value 0.326 is used in all the calculations in this paper.

\section{CAPACITY ANALYSIS}

In a speech-oriented DS CDMA system, after setting-up a call each user is allowed to transmit data continuously. A very important design issue for such a system is the user capacity. A service provider will be interested in the maximum number of users that can be served simultaneously by the system with a predefined performance in a certain geographic area. 
In a digital communication system such as DS CDMA, the bit error rate (BER) is an appropriate performance measure. BER calculations offer the possibility for detailed system considerations but often require complex models that can sometimes disturb the basic understanding of the system. In order to obtain a general idea about the influence of power control, sectorization, and voice activity on the capacity of a cellular DS CDMA system, a simplified model is proposed in this paper. Note that for a more detailed system investigation it is better to rely on BER models since the simplified model does not consider the effects of multipath in general.

The performance model used in this paper is based on a minimum required signal-to-interference ratio at the receiver in order to obtain a prespecified bit error rate performance. In [2] it was mentioned that irrespective of modulation method, the minimal signal to interference ratio after correlation for a bit error rate better than $10^{-3}$ is $E_{b} / N_{0}=5(7 \mathrm{~dB})$. The threshold value $\gamma$ for the signal-to-interference ratio before correlation is

$$
\gamma=\frac{1}{\mathrm{PG}} \cdot\left(\frac{E_{b}}{N_{0}}\right)_{\min }
$$

where PG is the processing gain, which is equivalent to the number of chips per bit in this case. In a system with multiple cells and (imperfect) sectorization, the signal-to-interference ratio at the receiver is

$$
\frac{C}{I}=\frac{P_{i}}{P_{I}\left(1+F_{m}\right) \cdot F_{s}}
$$

with

$P_{i}$ : Received power from desired terminal in home cell.

$P_{I}$ : Intra-cell interference power from users in home cell (single isolated cell without sectorization).

$F_{s}$ : Interference correction factor due to imperfect sectorization (8).

$F_{m}$ : Interference correction factor due to inter-cell interference (17).

The failure probability $P_{f}$ is given by

$$
P_{f}=\operatorname{Pr}\left(\frac{P_{i}}{P_{I}}<\gamma\left(1+F_{m}\right) \cdot F_{s}\right) .
$$

In the case of imperfect power control, the received power $P_{i}$ from each mobile is modeled as a log-normal variable with zero logarithmic mean and standard deviation $\sigma$. The pdf of the total interference signal produced by $(k-1)$ other active terminals is approximated by a lognormal variable with logarithmic mean $m_{I}(k-1)$ and standard deviation $\sigma_{I}(k-1)$ ((6) and (7), respectively) computed according to the method of Fenton [10]. After some mathematical manipulations the failure probability for $k$ active users can be written as

$$
P_{f}(k)=\frac{1}{2}+\frac{1}{2} \operatorname{erf}\left(\frac{\ln \left[\gamma\left(1+F_{m}\right) \cdot F_{s}\right]-m_{I}(k-1)}{\sqrt{2\left(\sigma^{2}+\sigma_{I}^{2}(k-1)\right)}}\right)
$$

where erf $(\cdot)$ is the error function, $\gamma$ is the threshold ratio for the signal-to-interference ratio, $F_{m}$ is the interference correction factor due to multiple cell interference, $F_{s}$ is the interference correction factor due to sectorization and $m_{I}(k-1)$ and $\sigma_{I}(k-1)$ are the logarithmic mean and the logarithmic standard deviation respectively of the total power received from $(k-1)$ users in case of imperfect power control with individual power control error $\sigma$. Assuming that there are $n$ users per cell ( $n \geq k$ ), each with voice activity $a$, then the failure probability is

$$
P_{f}^{a}(n)=\sum_{k=1}^{n} P_{f}(k) \cdot\left(\begin{array}{l}
n \\
k
\end{array}\right) a^{k}(1-a)^{n-k} .
$$

The user capacity is now defined as the maximum number of users per cell with voice activity $a$ such that the failure probability is less than 0.01 .

\section{ThroughPut and Delay Analysis}

For transmission of computer data, a packet communications schedule can be more efficient than using a circuit switched protocol. In this section we consider a slotted system, implying that that each user is allowed to transmit packets only at fixed time instants. In a packet network, throughput and delay are appropriate parameters, rather than maximum user capacity. The throughput determines the average number of successfully received packets per time slot, given a certain amount of traffic. For a certain amount of throughput it is important to know what will be the average delay of a packet. The throughput is defined as the average number of successfully received packets per time slot:

$$
S=\sum_{k=1}^{N} k P_{t}(k) P_{s}(k)
$$

where $N$ is the number of users per cell, $P_{s}(k)$ is the packet success probability and $P_{t}(k)$ is the probability of a packet transmitted with $k-1$ other packets.

It is assumed that a total of $n_{f}$ independent failures can occur within one time slot. This number in general depends on the length of a time slot $T_{\text {slot }}$ and the average time between two failures $T_{\mathrm{av}}$ and is defined as:

$$
n_{f} \stackrel{\wedge}{=} \frac{T_{\text {slot }}}{T_{\mathrm{av}}} .
$$

Now the packet success probability $P_{s}(k)$ defined as the probability of a test packet not being destroyed by $(k-1)$ interfering packets is

$$
P_{s}(k)=\left(1-P_{f}(k)\right)^{n_{f}}
$$

with $P_{f}(k)$ being the failure probability given by (21). Although it is difficult to implement power control to packet transmission due to short packet duration, the present analysis is based on power control for simplicity.

Because the number of simultaneous terminals is limited, the offered traffic is assumed to have a binomial distribution. The probability of a packet transmitted with $k-1$ other packets $P_{k}$ is then given by

$$
P_{t}(k)=\left(\begin{array}{c}
N \\
k
\end{array}\right)\left[\frac{G}{N}\right]^{k}\left[1-\frac{G}{N}\right]^{N-k}
$$

where $G$ is the average number of offered packets per time slot. Here it is assumed that the offered traffic consists of new packets and retransmission packets. 


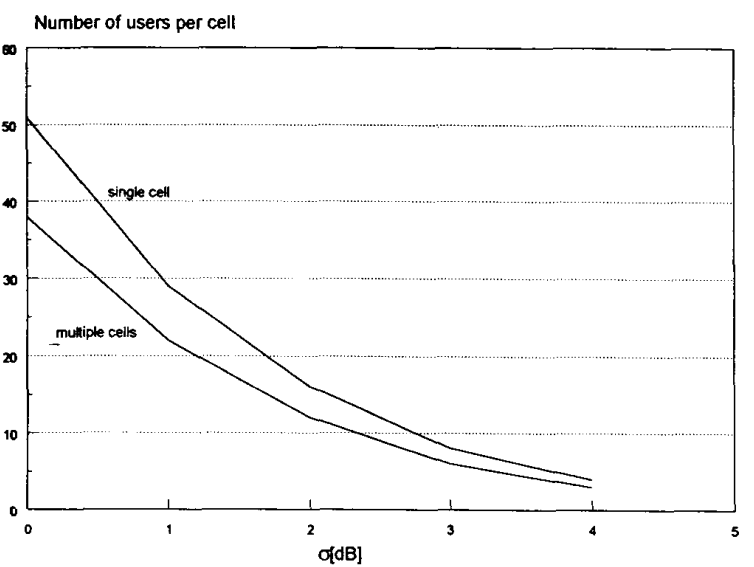

Fig. 5. Capacity as function of power control imperfection for a single cell and multiple cell system. Processing gain $P G=255$, no voice activity monitoring $(a=1)$, no sectorization $(D=1)$.

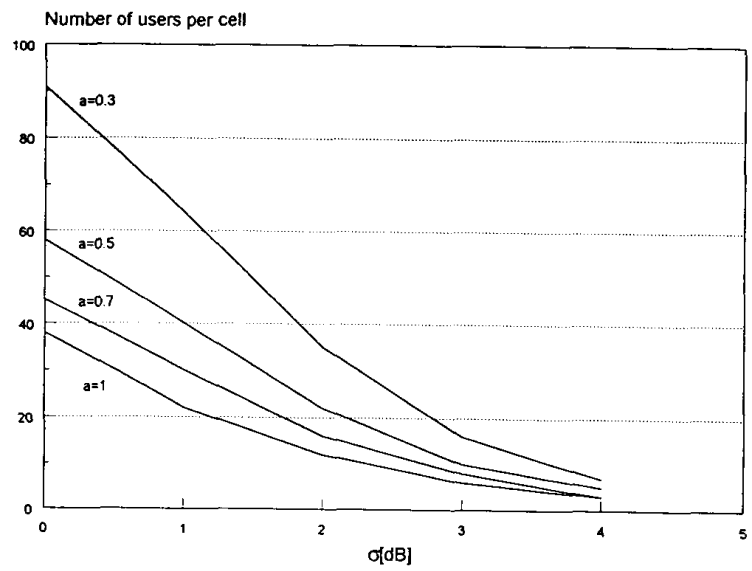

Fig. 6. Capacity as a function of power control imperfection for several values of the voice activity factor (a) Processing gain $\mathrm{PG}=255$, no sectorization $(D=1)$, multiple cells.

Another important performance parameter for a slotted packet network is the delay, which is defined as the average time between the generation and successful reception of a packet. Assuming a positive acknowledgment scheme and no transmission errors in the acknowledgment packets, the expression for the delay in a slotted DS CDMA system is [11]-[13]:

$$
D=1.5+\left[\frac{G}{S}-1\right] \cdot(\lfloor\delta+1\rfloor+1)
$$

where $\delta$ is the mean of the retransmission delay, which is uniformly distributed over the range from which the retransmission delay is selected, $S$ is the throughput and $G$ is the offered traffic. Note that the delay in (27) is normalized on the slot time.

\section{RESULTS}

All figures presented is this section are valid for the reverse link. First the results are given for the capacity, i.e., the maximum number of users per cell. For all results, arbitrary

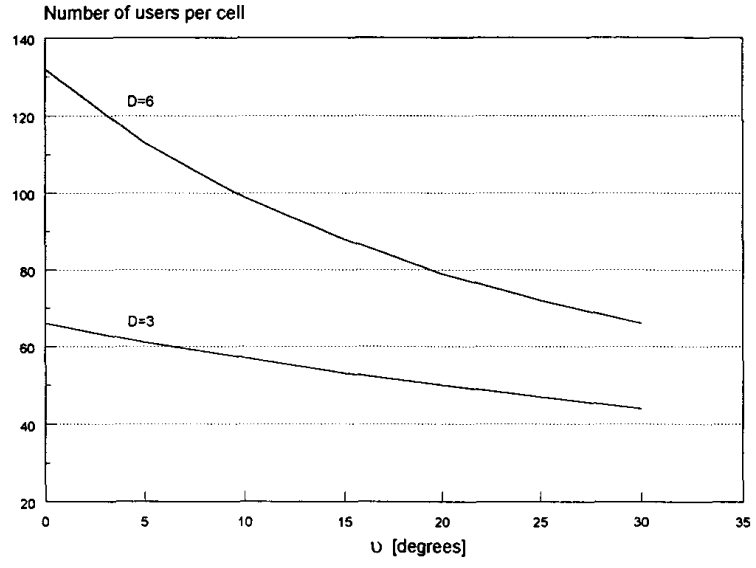

Fig. 7. Capacity as a function of overlap angle for sectorization with sectorization degree $D=3$ and $D=6$. Processing gain $\mathrm{PG}=255$, power control imperfection $\sigma=1 \mathrm{~dB}$, no voice activity monitoring $(a=1)$, multiple cells.

PN sequences with 255 chips per bit are assumed, implying a processing gain $P G=255$. Fig. 5 shows the influence of power control imperfections on the capacity for a DS CDMA system with only one cell and for a system with multiple cells. In the multiple cell case the inter-cell interference was modeled as a fraction of the intra-cell interference using (17) where we assumed a path loss law exponent $\beta=4$. In case of perfect power control, the maximum capacity shows a decrease of $25 \%$ when considering multiple cells instead of a single cell. For lower values of $\beta$ the decrease in capacity due to multiple cell interference will be higher. Fig. 5 also shows that the system is very sensitive to power control errors; a power control error of only $1 \mathrm{~dB}$ gives a capacity reduction of about $60 \%$. A power control error of $1 \mathrm{~dB}$ is interpreted as the standard deviation of $1 \mathrm{~dB}$ around the desired power level. In [5] it was concluded that a standard deviation of $2.5 \mathrm{~dB}$ causes a reduction in capacity of only $20 \%$. In [5], however, it was assumed that an outage occurs if the interference power density exceeds the background noise level by $10 \mathrm{~dB}$ whereas in our paper an outage occurs if the signal-to-noise-ratio after correlation exceeds a predefined value $\left(E_{b} / N_{0}=5(7 \mathrm{~dB})\right)$. This explains the difference in capacity reduction. Fig. 6 shows that the capacity decreases as the voice activity factor increases. If the processing gain is increased, i.e., if the number of chips per bit is increased, then the maximum number of users increases at the cost of more required bandwidth (since the chip duration must decrease in order to keep the data rate fixed). As expected, the capacity increases with the increase in the degree of sectorization, which is shown in Fig. 7. Furthermore it can be seen from Fig. 7 that the capacity decreases as the overlap angle due to imperfect sectorized antennas increases. The model with the uniform antenna gain pattern and the overlap angle is a drastic simplification of real antenna gain patterns but it simplifies the calculations tremendously. It suggests to transform real antenna gain patterns into uniform patterns analogously to modeling thermal filtered noise by the noise equivalent bandwidth. 


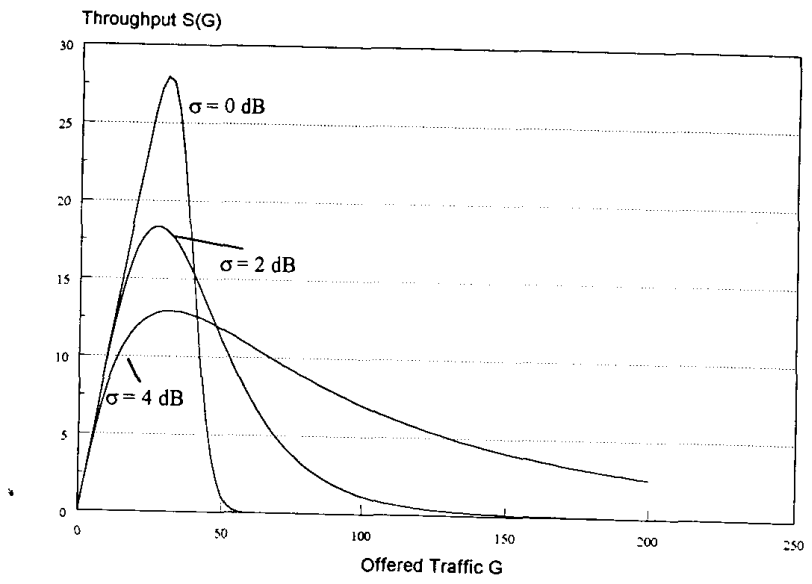

(a)

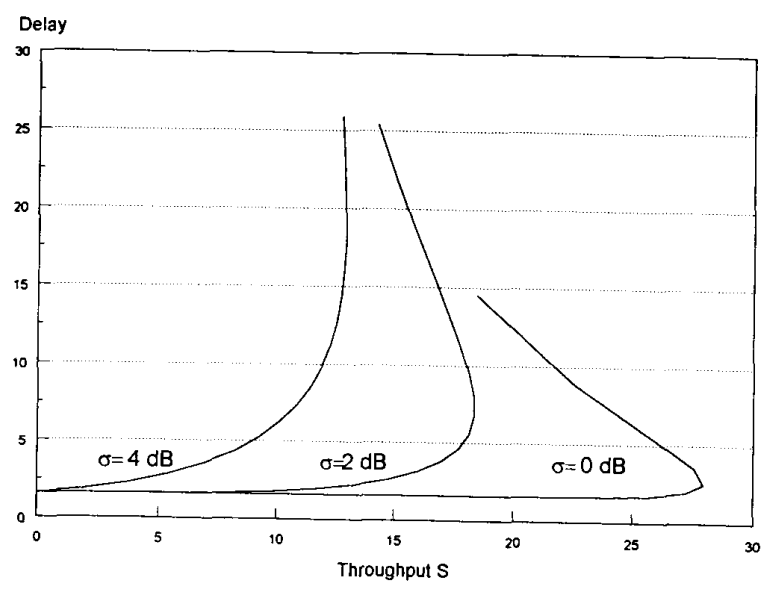

(b)

Fig. 8 . (a) Influence of power control error on throughput. Processing gain $\mathrm{PG}=255,200$ terminals per cell, and no sectorization. (b) Influence of power control error on the delay. Processing gain $P G=255,200$ terminals per cell, and no sectorization.

In a packet-oriented DS CDMA system the throughput and delay characteristics are more appropriate performance parameters than the maximum user capacity. For all the following figures it is assumed that on the average one fade per packet occurs $\left(n_{f}=1\right)$ and that the processing gain PG again is 255 . The throughput is given per cell and the delay is measured in time slot units. For the delay calculations we assumed a mean retransmission delay $\delta=10$ slot times. Fig. 8(a) and (b) shows the influence of power control imperfections on the throughput and delay, respectively. It is clear that again the performance decreases as the power control error increases. A decrease in performance implies here that the maximum throughput is lower and that the delay corresponding to a throughput value is higher. From Fig. 8(a) it is clear that for high traffic loads the throughput increases for higher power control errors. This can be explained by considering that in the case of perfect power control the failure probability always equals 1 if the number of users exceeds the maximum capacity. This is not the case if we have imperfect power control ( $\sigma \geq 0 \mathrm{~dB}$ ). Because of the tail of the log-normal distribution, there is always a probability that

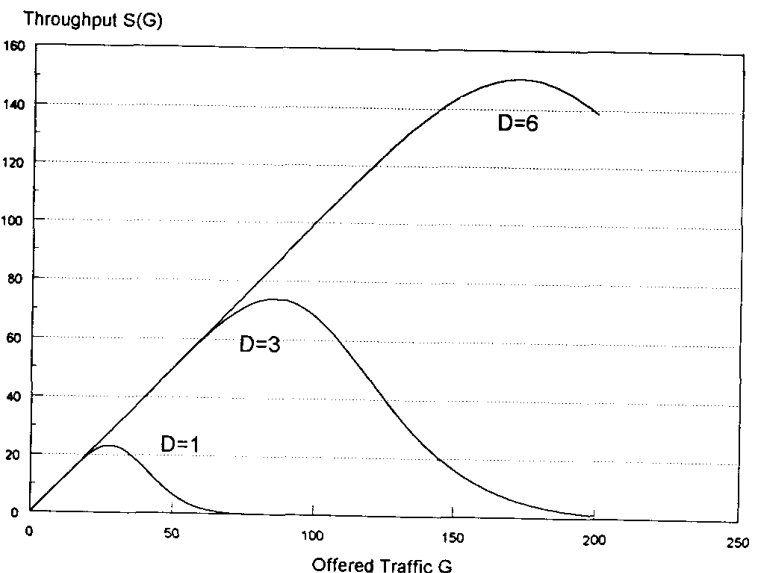

(a)

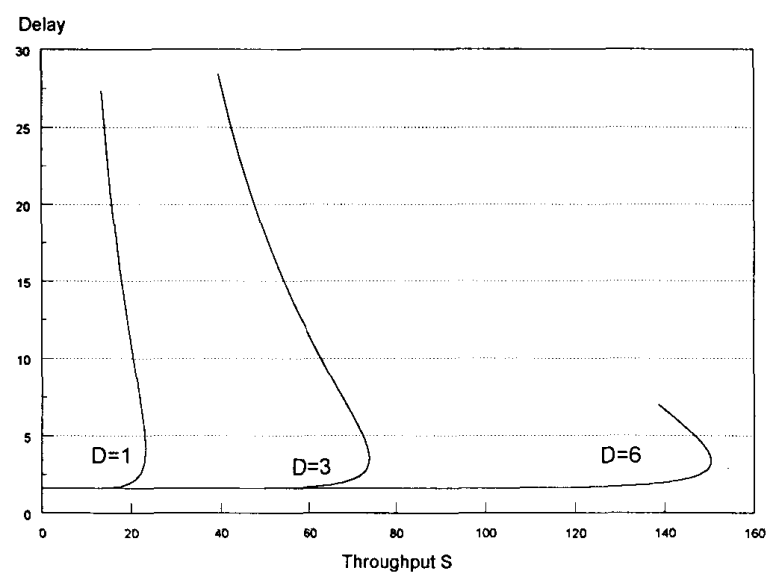

(b)

Fig 9 (a) Influence of sectorization on throughput. Power control error $\sigma=1 \mathrm{~dB}$, processing gain $\mathrm{PG}=255,200$ terminals per cell, and perfect sectorization. (b) Influence of sectorization on delay. Power control error $\sigma=1 \mathrm{~dB}$, processing gain $\mathrm{PG}=255,200$ terminals per cell, and perfect sectorization.

a few users are successful although the total number of users exceeds the maximum capacity. Then the failure probability decreases as the variance in the received power due to power control errors increases. Note that this effect occurs in the overload region.

The influence of sectorization (perfect) on the throughput and delay is depicted in Fig. 9(a) and (b). As expected, the performance increases with increasing number of sectors. From Fig. 9(a) it can be seen that the maximum throughput is better than proportional, i.e., the maximum throughput for $D=3$ and $D=6$ is higher than $3 . S$ at $D=1$ and 6.S at $D=1$ respectively. In order to explain this it is first of all important to observe that the average number of offered packets at the maximum throughput is below the maximum capacity (maximum number of simultaneously transmitting users). This is caused by the fact that already for values of average offered traffic less than the maximum capacity there is a probability that the actual number of offered packets exceeds the maximum capacity. The maximum capacity of the cell is of course exactly proportional to the 


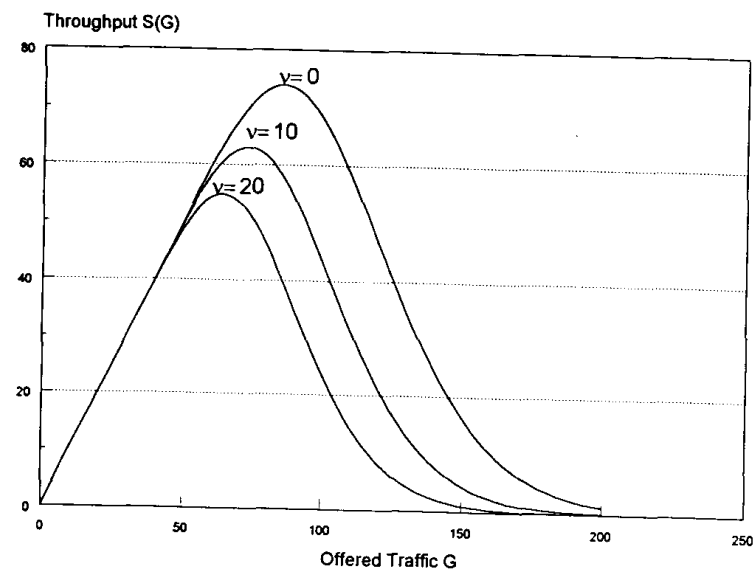

(a)

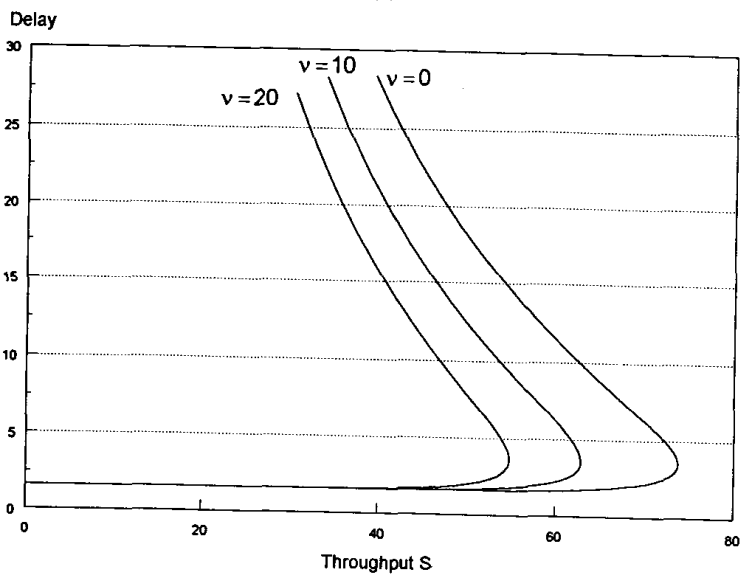

(b)

Fig. 10. (a) Influence of overlap angle (in degrees) on throughput. Power control error $\sigma=1 \mathrm{~dB}$, processing gain $\mathrm{PG}=255,200$ terminals per cell, and $D=3$ sectors. (b) Influence of overlap angle (in degrees) on delay. Power control error $\sigma=1 \mathrm{~dB}$, processing gain $\mathrm{PG}=255,200$ terminals per cell, and $D=3$ sectors.

number of sectors in the cell. Since the total user population for each cell is the same for the sectorization schemes we considered $(N=200)$, the expression for the probability of having $k$ simultaneous packets in one entire cell $\left(P_{t}(k)\right)$ is the same. The probability of increasing a maximum user capacity of 50 users is then higher than the probability of increasing a maximum user capacity of 150 users. This implies that the difference between the maximum offered traffic and the maximum capacity decreases with an increasing degree of sectorization and explains that the maximum throughput increases more than proportionally in our model.

Finally, Fig. 10(a) and (b) shows the influence of antenna imperfections on the throughput and delay of a slotted DS CDMA system. The performance decreases as the overlap angle due to imperfect directional antennas increases.

\section{CONCLUSION AND RECOMMENDATIONS}

A general model has been presented in this paper that is suitable to evaluate the reverse link performance of a cellular DS CDMA system in terms of maximum user ca- pacity, throughput, and delay. The multiple cell interference is modeled analytically for an environment with path loss law exponent $\beta$. In order to keep the model comprehensible and to investigate global effects of power control, sectorization and voice activity monitoring, effects of shadowing, and multipath propagation have not been considered. Results have been presented in this paper for a path loss law exponent $\beta=4$. Voice-oriented as well as data-oriented systems were evaluated and the effects of imperfect power control and imperfect sectorization were studied. It was shown that a power control error of $1 \mathrm{~dB}$ leads to a capacity loss of 50 to $60 \%$. Furthermore, an overlap degree of $15^{\circ}$ due to imperfect sectorization in a system with three sectors per cell causes a capacity reduction of $20 \%$. This decrease in performance can not only be seen due to the lower maximum user capacity but also due to lower maximum throughput and higher delay.

The model described in this paper is appropriate for investigating general system properties in a very clear way and can be used to obtain a first general idea of the effects of voice activity monitoring, power control, and sectorization on the performance. In order to obtain more detailed information, however, it is recommended that shadowing effects, multipath statistics, and consideration of BER performance be included. This will be the subject of future study.

\section{REFERENCES}

[1] R. Prasad, M. G. Jansen, and A. Kegel, "Capacity analysis of a cellular direct sequence code division multiple access system with imperfect power control," IEEE Trans. Commun., vol. E76-B, no. 8, pp. 894-905, Aug. 1993.

[2] _ _ "Effect of imperfect power control on a cellular code division multiple access system," Elect. Lett., vol. 28, no. 9, pp. 848-849, Apr. 23, 1992.

[3] W. C. Y. Lee, "Overview of cellular CDMA," IEEE Trans. Commun., vol. 40, pp. 291-302, May 1991.

[4] K. S. Gilhousen, J. M. Jacobs, R. Padovani, A. J. Viterbi, L. A. Weaver, and C. E. Wheatly, III, "On the capacity of a cellular CDMA system," IEEE Trans. Commun., vol. 40, pp. 302-312, May 1991.

[5] A. M. Viterbi and A. J. Viterbi, "Erlang capacity of a power controlled CDMA system," IEEE J. Select. Areas Commun., vol. 11, pp. 892-899, Aug. 1993.

[6] A. J. Viterbi, A. M. Viterbi, and E. Zehavi, "Performance of power controlled wideband terrestrial digital communication," IEEE Trans. Commun., vol. 41, pp. 559-569, Apr. 1993.

[7] H. J. Braun, G. Cosier, D. Freeman, A. Gilloire, D. Sereno, C. B. Southcott, and A. Van der Krogt, "Voice control of the Pan-European digital mobile radio system," CSELT Tech. Rep., vol. XVIII, no. 3, pp.183-187, June 1990.

[8] M. G. Jansen and R. Prasad, "Throughput analysis of a slotted CDMA system with imperfect power control," in Inst. Elect. Eng. Colloquium on Spread Spectrum Techniques for Radio Commun. Syst., London, U.K., Apr. 27, 1993, pp. 8.1-8.4

[9] K. I. Kim, "CDMA cellular engineering issues," IEEE Trans. Vehicular Technol., vol. 42, pp. 345-349, Aug. 1993.

[10] L. F. Fenton, "The sum of a log-normal probability distribution in scattered transmission systems," IRE Trans., vol. C5-8, pp. 57-67, Mar. 1960.

[11] L. Kleinrock and F. A. Tobagi, "Packet Switching in radio channels: Part I-Carrier sense multiple access modes and their throughput-delay characteristics," IEEE Trans. Commun., vol. COM-23, Dec. 1975.

[12] R. Prasad, C. A. F. J. Wijffels, and K. L. A. Sastry, "Performance analysis of slotted CDMA with DPSK modulation diversity and BCHCoding in indoor radio channels," $A E \ddot{U}$, vol. 46 , no. 6, pp. 375-382, Nov. 1992.

[13] C. A. F. J. Wijffels, H. S. Misser, and R. Prasad, "A micro-cellular CDMA system over slow and fast Rician fading channels with forward error correcting coding and diversity," IEEE Trans. Vehicular Technol., vol. 42, pp. 570-580, Nov. 1993. 


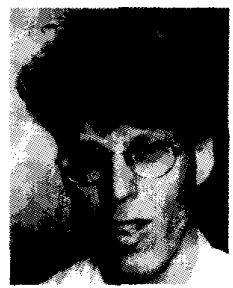

Michel G. Jansen received the Ir (M.Sc. E.E.) degree in electrical engineering from Delft University of Technology. The Netherlands in 1992. His dissertation was on the effects of imperfect power control on the capacity of DS CDMA networks.

After his graduation he joined the Telecommunications and Traffic-Control Systems Group of Delft University of Technology as a post-graduate student, where he is participating in the research program on CDMA radio networks.

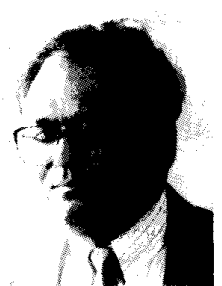

Ramjee Prasad (M'88-SM'90) received the B.Sc Eng. degree from Bihar Institute of Technology. Sindri, India, and the M.Sc. Eng. and Ph.D. degrees from Birla Institute of Technology (BIT), Ranchi. India, in 1968, 1970, and 1979, respectively.

He joined BIT as a Senior Research Fellow in 1970 and became an Associate Professor in 1980. During 1983-1988 he was with the University of Dar es Salaam (UDSM), Tanzania, where he became a Professor in Telecommunications at the Department of Electrical Engineering in 1986. Since February 1988, he has been with the Telecommunications and Traffic Control Systems Group, Delft University of Technology, The Netherlands, where he is actively involved in the area of mobile, indoor, and personal radio communications. While he was with BIT, he supervised many research projects in the area of microwave and plasma engineering. At UDSM, he was responsible for the collaborative project "Satellite Communications for Rural Zones" with Eindhoven University of Technology, The Netherlands. He has published more than 200 technical papers. His current research interest lies in packet communications, adaptive equalizers, spread-spectrum CDMA systems, and multimedia communications. He has also presented tutorials on mobile and indoor radio communications at various universities, technical institutions, and IEEE conferences. He is also a member of a working group of European cooperation in the field of scientific and technical research (COST-231) project "Evolution of Land Mobile Radio (including personal) Communications" as an expert for The Netherlands.

Dr. Prasad is listed in Who's Who in the World. He was Organizer and Interim Chairman of the IEEE Vehicular Technology/Communications Society Joint Chapter, Benelux Section. Now he is the elected Chairman of the same chapter. $\mathrm{He}$ is also founder of the IEEE Symposium on Communications and Vehicular Technology (SCVT) in the Benelux and was Symposium Chairman of SCVT'93. He has served as a member of advisory and program committees of several IEEE international conferences. He is one of the Editors-in-Chief of a new joumal on Wireless Personal Communications and is also a member of the editorial boards of other international journals including IEEE Communications Magazine. He was the Technical Program Chairman of the PIMRC'94 International Symposium held in The Hague, The Netherlands. September 19-23, 1994 and also of the Third Communication Theory Mini-Conference in conjunction with GLOBECOM`94, San Francisco, CA, November 27-30, 1994. He is a Fellow of the Institute of Electrical Engineers, a Fellow of the Institution of Electronics and Telecommunication Engineers, and a Member of the New York Academy of Sciences and The Netherlands Electronics and Radio Society. 